\title{
4. On a Conjecture of Roth \\ and Some Related Problems I
}

P. Erdő́s, A. Sárkōzy ${ }^{(1)}$, V.T. Sós ${ }^{(2)}$

\section{Introduction}

Let $N$ denote the set of positive integers and put $\{1, N]=\{1, \ldots, N\}$. We use $|S|$ to denote the cardinality of the finite set $S$. If $S$ is a given set and $A_{1}, \ldots, A_{k}$ are subsets of $S$ with

$$
S=\cup_{i=1}^{k} A_{i}, \quad A_{i} \cap A_{j}=\emptyset \text { for } i \neq j,
$$

then $\left\{A_{1}, \ldots, A_{k}\right\}$ will be called a $k$-partition (or $k$-colouring) of $S$, and the subsets $A_{1}, \ldots, A_{k}$ will be referred to as classes. Let $f: N^{t} \rightarrow \mathcal{N}$ be a given function. If

$$
n=f\left(a_{1}, \ldots, a_{t}\right)
$$

with $a_{1}, \ldots, a_{t}$ belonging to the same class, then this will be called a monochromatic representation of $n$ in the form (1)

For a fixed $k$-partition and $f$ we consider the set of integers, which have a monochromatic representation and investigate

a) how dense this set must be?

b) for which $S \subseteq N$ it must contain an element in $S$ ?

c) what sort of structural properties this set has?

We consider first the case $f\left(x_{1}, x_{2}\right)=x_{1}+x_{2}$.

Let $C$ resp. $C^{2}$ denote the set of integers resp. the set of even integers which have a monochromatic representation in the form

$$
n=a_{1}+a_{2} \text { with } a_{1} \neq a_{2}
$$

$$
\text { Put } \mathrm{C}_{M}=\mathrm{C} \cap[1, M] \text { and } \mathrm{C}_{M}^{2}=\mathrm{C}^{2} \cap[1, M] \text {. }
$$

(1),(2) Research partially supported by Hungarian National Foundation for Scientific Research grant no. 1811 
K.F. Roth conjectured isee $[4]$ and [9], p.112) that there is an absolute constant $c>0$ such that for an arbitrary $k$-partition

(3)

$$
\left|\mathrm{C}_{M}\right|>c M \text {. }
$$

(Note that if also $a_{1}=a_{2}$ is allowed, then this is trivial.)

We prove this conjecture in a sharper and more general form. We study some related problems too.

The Case $f\left(x_{1}, x_{2}\right)=x_{1}-x_{2}$

Theorem 1.

(i) To every $k \geq 2$ there exists an $M_{0}(k)$ such that for an arbitrary $k$-partition of $\mathrm{N}$

$$
\left|C_{M}^{2}\right|>\frac{M}{2}-3 M^{1-2^{-k-1}} \text { if } M>M_{0}(k) \text {. }
$$

Moreover

(ii) For every 2-partition

$$
\left|C_{M}^{2}\right|>\frac{M}{2}-\left(\log \left(\frac{1+\sqrt{5}}{2}\right)\right)^{-1} \log M
$$

(iii) There is a 2-partition so that

$$
2^{n} \notin C^{2} \text { for } n \in \mathcal{N}
$$

Proof.

(i) The proof will be based on the following

Lemma 1. If $d \in N, M>M_{0}(d), B \subseteq[1, M]$ and

$$
|B|>3 M^{1-2^{-\alpha}}
$$

then there exist positive integers $u, v_{1}, \ldots, v_{d}$ such that $v_{i} \neq v_{j}$ for $i \neq j$ and all the $2^{d}$ sums

$$
u+\sum_{i=1}^{d} \varepsilon_{i} v_{i}, \quad \varepsilon_{i} \in\{0,1\}
$$

belong to $B$. 
This is a density version of Hilbert's lemma [10] (which is considered as the first Ramsey-type result). See aiso [8]. It can be proved similariy to Lemma 7 in [14] (see also [3] and [20]). However for the sake of completeness, we give the proof here.

Proof of Lemma 1. It suffices to show the existence of sets $B_{0}, B_{1}, \ldots, B_{d}$ and distinct positive integers $v_{1}, v_{2}, \ldots, v_{d}$ such that

$$
B_{0}=B \text {, }
$$

and

$$
B_{j} \cup\left\{b+v_{j}: b \in B_{j}\right\} \subset B_{j-1} \text { for } j=1,2, \ldots, d
$$

In fact, if $B_{0}, B_{1}, \ldots, B_{d}, v_{1}, \ldots, v_{d}$ satisfy these conditions and $u \in B_{d}$, then by (9) and (10), $u+\sum_{i=1}^{d} \varepsilon_{i} v_{i} \in B$ for $\varepsilon_{i}=0$ or 1 , while (7) and (11) imply that $B_{d}$ is not empty. This then will complete the proof of Lemma 1 .

We are going to construct $B_{0}, B_{1} \ldots, B_{d}, v_{1}, \ldots, v_{d}$ recursively. Let $B_{0}=B$. Assume now that $0 \leq j \leq d-1$ and, in the case $j>0, v_{1}, \ldots, v_{j}$ have already been defined. For $1 \leq h \leq M-1$, let $f\left(B_{j}, h\right)$ denote the number of solutions of

$$
b-b^{\prime}=h, \text { where } b, b^{\prime} \in B_{j} .
$$

Then in order to define $B_{j+1}$ and $v_{j+1}$, we need an estimate for

$$
L=\max f\left(B_{j}, h\right)
$$

where the maximum is over all $h$ with $h \in[1, M], h \notin\left\{v_{1}, v_{2}, \ldots, v_{j}\right\}$.

Clearly, for all $h$ we have $f\left(B_{j}, h\right) \leq\left|B_{j}\right|$. Also

$$
\sum_{h=1}^{M-1} f\left(B_{j}, h\right)=\left(\begin{array}{c}
\left|B_{j}\right| \\
2
\end{array}\right)
$$

since $b-b^{\prime} \in[1, M]$ for any pair $b, b^{\prime} \in B_{j}$ with $b>b^{\prime}$. If we majorize $f\left(B_{j}, h\right)$ by $\left|B_{j}\right|$ for $h \in\left\{v_{1}, v_{2}, \ldots, v_{j}\right\}$ and by $L$ otherwise, (12) implies

$$
\left(\begin{array}{c}
\left|B_{j}\right| \\
2
\end{array}\right) \leq j\left|B_{j}\right|+(M-1-j) L \leq j\left|B_{j}\right|+L M,
$$

so that

$$
L>\frac{1}{2 M}\left(\left|B_{j}\right|^{2}-\left|B_{j}\right|-2 j\left|B_{j}\right|\right)=\frac{\left|B_{j}\right|}{3 M}\left(\frac{3}{2}\left|B_{j}\right|-\frac{3}{2}-3 j\right) .
$$


From (7) and (11), we have (for $M$ larger than some absolute and computable constant)

$$
\begin{gathered}
\left|B_{j}\right| \geq|B|^{2^{j}}(3 M)^{-\left(2^{j}-1\right)}>\left(3 M^{1-2^{-d}}\right)^{2^{j}}(3 M)^{-\left(2^{j}-1\right)}= \\
=3 M^{1-2^{j-d}} \geq 3 M^{1-2^{-i}}>3+6 d>3+6 j,
\end{gathered}
$$

so that (11) and (13) imply

$$
L>\frac{\left|B_{j}\right|}{3 M} \cdot\left|B_{j}\right| \geq \frac{1}{3 M}\left(|B|^{2^{j}}(3 M)^{\left(2^{j}-1\right)}\right)^{2}=
$$

$$
=|B|^{2^{j+1}}(3 M)^{-\left(+2^{j+1}-1\right)} .
$$

Let $v_{j+1} \in\left[1, M \backslash \backslash\left\{v_{1}, v_{2}, \ldots, v_{j}\right\}\right.$ denote an integer for which the maximum in the definition of $L$ is attained, i.e., $L=f\left(B_{j}, v_{j+1}\right)$ with $v_{j+1} \notin$ $\left\{v_{1}, v_{2}, \ldots, v_{j}\right\}$, and let

$$
B_{j+1}=\left\{b: b \in B_{j}, b+v_{j+1} \in B_{j}\right\} .
$$

Thus (10) holds for $j+1$ in place of $j$ and since $\left|B_{j+1}\right|=L,(14)$ implies that (11) holds also for $j+1$ in place of $j$. This completes the proof of the existence of $B_{0}, B_{1}, \ldots, B_{d}, v_{1}, \ldots, v_{d}$ with the desired properties, so that Lemma 1 is proved.

To prove the first statement in Theorem 1, we assume that there are more than $3 M^{1-2^{-k-1}}$ even integers not exceeding $M$ which do not have a monochromatic representation in the form (2); let us denote the set of these integers by 8 . Then (3) holds with $k+1$ in place of $d$, thus if $M$ is sufficiently large, then by Lemma 1 there exist positive integers $u, v_{1}, v_{2}, \ldots, v_{k+1}$ such that all the sums

$$
u+\sum_{i=1}^{k+1} \varepsilon_{i} v_{i} \text { where } \varepsilon_{i}=0 \text { or } 1
$$

belong to $B$. Then

$$
u=u+\sum_{i=1}^{k+1} 0 \cdot v_{i} \in B
$$

and since $\hat{b}$ consists of even numbers, thus also $u=2 z$ is even. The integers $z+v_{1}, z+v_{2}, \ldots, z+v_{k+1}$ are distinct, thus by the pigeon hole principle, there exist $1 \leq i<j \leq k+1$ such that $a_{1}=z+v_{i}$ and $a_{2}=z+v_{j}$ belong to the same class. Then $a_{1}+a_{2}$ is a monochromatic sum with $a_{1} \neq a_{2}$, and 


$$
a_{1}+a_{2}=\left(z+v_{i}\right)+\left(z+v_{j}\right)=2 z+v_{i}+v_{j}=u+v_{i}+v_{j}
$$

But this contradicts the definition of $B$, and the proof of the first half of Theorem 1 is completed.

(ii) Let $B=\left\{b_{1}, b_{2}, \ldots, b_{t}\right\}$ (where $b_{1}<b_{2}<\ldots<b_{t}$ ) denote the set of those even integers not exceeding $2 M$ which do not have a monochromatic representation in the form (2).

Suppose

$$
b_{j+2}<b_{j} \dot{+} b_{j+1}
$$

for some $j$. Then there are positive integers $x, y, z$ for which

$$
\begin{aligned}
& x+y=b_{j} \\
& x+z=b_{j+1} \\
& y+z=b_{j+2}
\end{aligned}
$$

At least two of these numbers belong to the same class. This contradicts to the definition of $B$. Hence for every $j$

$$
b_{j+2} \geq b_{j}+b_{j+1}
$$

which proves (ii)

To prove (iii) we define the set $A_{1}$ recursively. Let $1 \in A_{1}$. If $A \cap\left[1,2^{k-1}\right]$ has been defined, then let $2^{k} \in A_{1}$ and for $2^{k-1}<n<2^{k}, \quad n \in A_{1}$ iff $2^{k}-n \notin A_{1} \cap\left[1,2^{k-1}\right]$. Furthermore let $A_{2}=\mathcal{N} \backslash A_{1}$. Then obviously $2^{n} \notin \mathrm{C}$ for $n=1,2, \ldots$.

Observe that $\left|\mathrm{C}_{M}\right|$ need not be much greater then $\left|\mathrm{C}_{M}^{2}\right|$ as the following example shows: $A_{1}=\{2 j-1: j \in N\}, \quad A_{2}=\{2 j: j \in N\}$. However the situation is different for $k \leq 3$ and for $k \geq 4$.

Theorem 2.

(i) There is an absolute constant $C$ so that if $k \leq 3$ then at any $k$-partition

$$
\left|\mathrm{C}_{M}\right| \geq\left[\frac{M}{2}\right]-1 \quad \text { if } \quad M>C \text {. }
$$

(ii) If $k \geq 4$, there exists a $k$-partition such that

$$
\left|\mathrm{C}_{M}\right|<\frac{M}{2}-c k \log M,
$$

where $c$ is an absolute constant.

Proof of (i). Case $k=2$.

Without loss of generality we can assume that $x \in A_{1}$ for $1 \leq x \leq a$ and $a+1 \in \mathcal{A}_{2}$.

Then $y \in \mathrm{C}$ for $3 \leq y \leq 2 a-1$. On the other hand for every $y>0$ either $y+a \in \mathrm{C}$ or $y+a+1 \in \mathrm{C}$. 
Case $k=3$

Suppose $2 x-1 \in A_{1}$ if $1 \leq x \leq a$ and $2 a+1 \in A_{2}$. Then

$$
2 y \in \mathrm{C} \quad \text { if } \quad 2 \leq y \leq 2 a .
$$

We may assume that there is an $n>2 a$ such that

$$
2 n \notin C \text { and } 2 n-1 \notin C
$$

and

$$
\left|C_{2 n}\right|<\left[\frac{M}{2}\right]
$$

Case $12 n \leq 6 a$. Put $2 n=4 a+2 t, \quad(t \leq a)$. First we prove

$$
2 a+2 \subseteq A_{2} \text {. }
$$

Namely if $2 a+2 \in A_{1}$, then $2 x-1+2 a+2 \in \mathrm{C}$ for $1 \leq x \leq a$. Hence

$$
\left|\mathrm{C}_{2 n}\right|>2 a+a
$$

which contradicts (21).

Now suppose $2 a+2 \in A_{3}$. Then $2 n-(2 a+2)=2 a+2 t-2 \in A_{1} \cup A_{2}$. In case $2 a+2 t-2 \in A_{1}$

$$
2 x-1+2 a+2 t-2 \in \mathrm{C} \text { for } 1 \leq x \leq a .
$$

This implies

$$
\mathrm{C}_{2 n} \geq 2 a+a
$$

which contradicts again to (21).

In case $2 a+2 t-2 \in A_{2}$

$$
2 n-1=(2 a+1)+(2 a+2 t-2) \in \mathrm{C}
$$

would follow, which contradicts to (20).

Thus $2 a+2 \in A_{2}$.

Consider now the integers in $[2 a+2,2 a+2 t\}$. For every $y, 0 \leq y \leq 2 t$

$$
2 a+y \in A_{3} \text { implies } 2 a+2 t-y \in A_{3} \text {. }
$$

Therefore at least $t$ integers in $[2 a+2,2 a+2 t]$ belong to $A_{1} \cup A_{2}$.

If there is an even $x \in A_{1} \cup[2 a+2,2 a+2 t]$, then

$$
4 a<x+2 v-1<4 a+2 t=n \text { for } 1 \leq v \leq a .
$$

Hence

$$
\left|\mathrm{C}_{2 n}\right|>2 a+a
$$

which contradicts (21).

If all the $t$ even integers in $[2 a+2,2 a+2 t]$ belong to $A_{2}$, then for $1 \leq u \leq$ $t-1$

$$
(2 a+2+2 u)+2 a+1 \in \mathrm{C}
$$


and

$$
(2 a+2+2 u)+2 a+2 \in \mathrm{C}
$$

This would imply

$$
\left|\mathrm{C}_{2 n}\right|>3 a \text {. }
$$

This finishes the case when $2 n \leq 6 a$.

Case $2 n>6 a$.

Since $2 n \notin C$, at least $\frac{n-2}{2}$ even numbers below $2 n$ are in $A_{1} \cup A_{2}$. Thus at least $\frac{n-2}{2}-a$ even numbers below $2 n-2 a$ are in $A_{1} \cup A_{2}$. Therefore at least $\frac{n-2}{4}-\frac{a}{2}>\frac{n-2}{12}$ are in $A_{1}$ resp. in $A_{2}$. Adding to these numbers $2 a-1$ or $2 a+1$ we gain $\frac{n-2}{12}$ odd numbers in $\mathrm{C}$. Hence by Theorem 1

$$
\mathrm{C}_{2 n}>n-6 n^{\frac{15}{18}}+\frac{n-2}{12}>n \quad \text { if }
$$

$n$ is large enough.

Proof of (ii). We may suppose that $k=4 \ell$ where $\ell$ is odd. Define $t_{0}$ by

$$
2^{t_{0}-i} \leq 2 \ell<2^{t_{0}}
$$

For $i=1,2, \ldots, \ell$ we are going to define subsets $A_{4 i-j}, j=0,1,2,3$ recursively. Let for $j=1,3$

$$
A_{4 i-j} \cap\left[1,2^{t_{0}}\right]=\left\{n: n \equiv i(\bmod \ell), n \equiv\left[\frac{j}{2}\right](\bmod 2)\right\} \cap\left[1,2^{t_{0}}\right]
$$

and

$$
A_{4 i-j} \cap\left[1,2^{t_{0}}\right]=\emptyset \quad \text { if } j=0,2 .
$$

Assume now that $A_{4 i-j} \cap\left[1,2^{t}\right]$ have been defined for $j=0,1,2,3, i=$ $1, \ldots, 2 \ell+1$. Let $r_{i}(t)$ defined by

$$
\text { : } \quad 2 i \equiv 2^{t+1}+r_{i}(t)(\bmod 2 \ell), \quad 0 \leq r_{i}(t)<2 \ell .
$$

Now we define $A_{4 i-j} \cap\left[2^{t}+1,2^{t+1}\right]$ in the following way: let $2^{t}<n \leq 2^{t+1}$. For $2^{t}<n<2^{t+1} n \in A_{4 i-3}$ iff $n$ is even and

$$
n \equiv i(\bmod \ell), \quad 2^{t+1}+r_{i}(t)-n \notin A_{4 i-3} \cap\left[1,2^{t}\right], 2 \mid n
$$

$n \in A_{4 i-2}$ iff $n$ is even and

$$
n \equiv i(\bmod \ell), \quad n \notin A_{4 i-3},
$$

$n \in A_{4 i-1}$ iff $n$ is odd and

$$
n \equiv i(\bmod \ell), 2^{t+1}+r_{i}(t)-n \notin A_{4 i-1} \cap\left[1,2^{t}\right], 2 \mid n
$$

$n \in A_{4 i}$ iff $n$ is odd and

$$
n \equiv i(\bmod \ell), n \notin A_{4 i-1},
$$

Then clearly the sets $A_{4 i-j}, \quad 1 \leq i \leq \ell, 0 \leq j \leq 3$ give a $4 \ell$-partition of $\mathcal{N}$. Furthermore it can be seen easily that all the monochromatic sums 
$a_{1}+a_{2}, \quad a_{1} \neq a_{2}$ are even and none of these sums is equal to a number of the form $2^{t}+2^{j}$ where $t>t_{0}$ and $0 \leq j \leq \ell-1$. This completes the proof of Theorem 2.

By Theorem 1, there are more than $\frac{M}{2}-c_{1} M^{1-2^{k-1}}$ integers in $[1, M]$ which have a monochromatic representation in the form (2), and by Theorem 2 , the number of these integers can be less than $\frac{M}{2}-c_{2} k \log M$. It follows from a resuit of.Erdős and Sárközy (Theorem 8 in [5]) that if $k \in N, M \in N, M>$ $M_{0}(k), t \in N$ and $M^{2 / 3}(\log M)^{2}<t \leq M$, then almost all the sets $B$ with $B \subset\{1, M],|B|=t$ are such that for every $k$-partition of $[1, M]$ there is (at least one) element in $B$ which has a monochromatic representation in the form (2). (In fact, the following sharper statement is true: almost all of these sets $B$ are such that for every $A$ with $A \subset\left[1, \frac{M}{2}\right]$ and $|A|>\frac{1}{k}[M / 2 !$, there is an element in $B$ which can be represented in the form (2) with $a \in A, a^{\prime} \in A$.) Ruzsa [16] proved that if $f(x) \rightarrow+\infty$, then there exists an infinite sequence $D$ of positive integers such that $D(x)=\sum_{\substack{d \leq x \\ d \in D}} \mid=O\left(f(x)(\log x)^{2}\right)$, and if $A$ is a sequence of positive integers with positive upper asymptotic density, then $D$ intersects the set of the integers of the form $a+a^{\prime}$ where $a \in A, a^{\prime} \in A$. These results suggest that the upper bound $\frac{M}{2}-c k \log M$ is closer to the truth than the lower bound.

Recently Balog, Fürstenberg, Sárközy, Stewart, Lagarias, Odlyzko, Schearer $[1],[7],[13],[14],[17],[18],[19]$ and others have studied the solvability of the equations

$$
\begin{aligned}
& a-a^{\prime}=x^{2} \\
& a-a^{\prime}=p-1 \\
& a+a^{\prime}=x^{2} \\
& a+a^{\prime}=p x, x^{n} \text { small" }(=0(1))
\end{aligned}
$$

with $a, a^{\prime} \in A$ where $A$ is a "dense" sequence of positive integers. These resuits and Hindman's theorem [2], [11] led us to consider the corresponding "monochromatic" questions.

Theorem 1 implies that e.g. the equations

$$
\begin{aligned}
& a_{1}+a_{2}=2 p \\
& a_{1}+a_{2}=p-1
\end{aligned}
$$

have monochromatic solutions with $a_{1} \neq a_{2}$.

Our result is not strong enough to obtain for arbitrary $k$ that

$$
a_{1}+a_{2}=x^{2}
$$

has a monochromatic solution with $a_{1} \neq a_{2}$. However a simple argument leads to 
Theorem 3. If $k \leq 3$, then for any $k$-partition of $N$ there are infinitely many squares in $\mathrm{C}$.

Proof. We use the following simple (and well known)

Lemma 2. For every $\varepsilon>0$ there are infinitely many integers $n$ so that

$$
n=x^{2}+y^{2}
$$

has at least three (in fact arbitrary many) integer solutions where

$$
x^{2}, y^{2} \in\left[\frac{n}{2}(1-\varepsilon), \frac{n}{2}(1+\varepsilon)\right] .
$$

Now let

$$
x_{1}^{2}+x_{6}^{2}=x_{2}^{2}+x_{5}^{2}=x_{3}^{2}+x_{4}^{2}
$$

with $x_{i} \in\left[\frac{n}{2}(1-\varepsilon), \frac{n}{2}(1+\varepsilon)\right], 1 \leq i \leq 6$.

Then an easy calculation shows, that the system

$$
\begin{aligned}
& u_{1}+u_{2}=z_{1}^{2} \\
& u_{3}+u_{4}=x_{5}^{2} \\
& u_{2}+u_{3}=x_{2}^{2} \\
& u_{1}+u_{4}=x_{5}^{2} \\
& u_{1}+u_{3}=x_{3}^{2} \\
& u_{2}+u_{4}=x_{4}^{2}
\end{aligned}
$$

in $u_{i}(1 \leq i \leq 4)$ has a solution in distinct positive numbers. Since at least two of the $u_{i}$ 's belong to the same class, one of the $x_{i}^{2}(1 \leq i \leq 6)$ squares must have a monochromatic representation.

If we have some information on the structure of the classes $A_{i}$ in the given partition then the lower bound given for the integers that have a monochromatic representation in form (2) can be sharpened. In fact we have

Theorem 4.

(i) For every $\varepsilon>0$ and $k$ there exists an $M_{0}(\varepsilon, k)$ such that if we have a $k$-partition of $\mathcal{N}$ where every class contains both even and odd integers then.

$$
\left|\mathrm{C}_{M}\right|>\left(\frac{1}{2}+\frac{1}{2 k}-\varepsilon\right) M \text { if } M>M_{0}(\varepsilon, k) .
$$

(ii) For every $k \in \mathcal{N}$ there is a $k$-partition of $\mathcal{N}$ so that every class contains both even and odd integers and

$$
\left|\mathrm{C}_{M}\right|<\left(\frac{1}{2}+\frac{1}{k}\right) M+1 .
$$




\section{Proof.}

(i) can be proved by the method used in the proof of Theorem 2 ,

(ii) follows from the following construction: for $i=1,2, \ldots, k$ let

$$
A_{i}=\{n: n \equiv 2 i(\bmod 2 k)\} \cup\{n: n \equiv 1-2 i(\bmod 2 k)\} .
$$

It is easy to see that this $k$-partition of $N$ has the desired properties.

The Case $f\left(x_{1}, x_{2}\right)=\left|r x_{1}+s x_{2}\right|$.

Let $r, s$ be integers. As before, let $\mathrm{C}$ denote the set of integers which have a monochromatic representation in the form

$$
n=\left|r a_{1}+s a_{2}\right| \text { with } a_{1} \neq a_{2} .
$$

Let $\mathrm{C}_{M}=: C \cap[1, M]$. The following result is merely a simple modification of Theorem 1.

Theorem 5. Let $r \neq 0, s \neq 0, r+s \neq 0$. Put $|r+s|=m$. For every $\varepsilon>0, k, r, s$ and for every $k$-partition

$$
\left|\mathrm{C}_{M}\right| \geq(1-\varepsilon) \frac{M}{m} \text {. }
$$

This can not be essentially improved, since choosing

$$
k=m \text { and } A_{i}=\{n: n \equiv i(\bmod m)\}, 1 \leq i \leq m
$$

only the muitiples of $m$ have a monochromatic representation in the form (22)

Note furthermore that Theorem 5 does not cover the case of the differences $a_{1}-a_{2}$. Namely, in this case the density of the integers having a monochromatic representation in the form (22) need not be greater than a positive absolute constant. To see this, let us consider a large integer $m$ and define the partition as in (23). Then only the multiples of $m$ have a monochromatic representation in the form (22) so that their density is $\frac{1}{m}$ which $\rightarrow 0$ if $m \rightarrow \infty$.

Proof. Assume that there are more than $\varepsilon \frac{M}{m}$ positive multiples of $m$ in $[1, M]$ which do not have a monochromatic representation in the form [22]. Then by Szemerédi's theorem $[20]$, for $M>M_{0}(k, \varepsilon, r, s)$ their set must contain an arithmetic progression of $2(|r|+|s|) k+1$ terms; let us write this arithmetic progression (all whose terms are multiples of $m$ ) in the form

(24) $u m-(|r|+|s|) k v, u m-((|r|+|s|) k-1) v, \ldots, u m+(|r|+|s|) k v$. 
Let us consider the integers $u, u+v, \ldots, u+k v$. By the pigeon hole principle, two of them, say $a_{1}=u+i v$ and $a_{2}=u+j v$ (where $i \neq j$ ) belong to the same class. Then

$$
r a_{1}+s a_{2}=r(u+i v)+s(u+j v)=(r+s) u+(r i+s j) v .
$$

Here we have

$$
|r i+s j| \leq i r|k+| s \mid k=(|r|+|s|) k .
$$

Since $|r+s|=m$ and all the numbers in (24) are positive, $\left|r a_{1}+s a_{2}\right|$ is equal to one of the numbers in (24). But this contradicts the fact that none of these numbers has a monochromatic representation in the form (22), and the proof is completed.

\section{Some Unsolved Problems}

Problem 1. Do there exist $\alpha$ and $\beta$ which depend only on $k$, so that for an arbitrary $k$-partition

$$
\left|\mathrm{C}_{M}\right|>\frac{M}{2}-(\log M)^{\alpha(k)}
$$

or even more $\left|C_{M}^{2}\right|>\frac{M}{2}-(\log M)^{\beta(k)}$.

Problem 2. Let $f(x)$ be a polynomial of integer coefficients such that 2 is a prime divisor of it. Is it true that for any $k$-partition for some $z$ (or for infinitely many $x$ )

$$
a_{1}+a_{2}=f(x)
$$

have a monochromatic solution with $a_{1} \neq a_{2}$ ?

Problem 3. Is it true that for every $k$-partition of $[1, M]$ aimost all the even integers $2 n$ in $[1, M]$ have more than $c(k) n$ monochromatic representations in form (2)? (Perhaps this holds with $c(k)=\frac{c_{1}}{k}$.)

Problem 4 a) For a given $k$-partition let $n_{1}<n_{1}<\ldots$ be the sequence of those integers which have a monochromatic representation in form (2). (C = $\left\{n_{i}\right\}$ ). What can be said about the structure of the sequence $\left\{n_{i}\right\}$ ? (For example it is easy to see that $\left|n_{i+1}-n_{i}\right|<2 k$.)

b) The complementary problem is to study the structure of the set $3=\mathcal{N}-\mathrm{C}$ (the set of those integers which do not have a monochromatic representation in form (2)).

Let $\mathcal{G}(N ; E)$ be the graph with edgeset $\{(x, y) \mid x+y \in 8, x, y \in \mathcal{N}\}$. Obviously at any $k$-partition the chromatic number of $\mathcal{G}(N ; E)$ is $\leq k$. Basically this was used in the proofs above. 
Problem 5. So far we have studied monochromatic representations in form (1) in the special case when $f\left(x_{1}, \ldots, x_{t}\right)$ is a linear polynomial and $t=2$. In the paper Erdös-Sárkōzy $[6]$ the case $f\left(x_{1}, x_{2}\right)=x_{1} x_{2}$ is considered.

What can one say on general polynomials $f\left(x_{1}, \ldots, x_{t}\right)$ (whose coefficients are integers)? What can be said in the most important special case when $f\left(x_{1}, x_{2}, \ldots, x_{t}\right)$ is of the form $g\left(x_{1}\right)+\ldots+g\left(x_{t}\right)$ ?

As Ruzsa [15] observed, if

$$
f\left(x_{1}, x_{2}, x_{3}, x_{4}\right)=x_{1}^{2}+x_{2}^{2}+x_{3}^{2}+x_{4}^{2}
$$

then for every $k$-partition

$$
\left|\mathrm{C}_{M}\right|>c(k) \cdot M
$$

and $\left|\mathrm{C}_{M}\right|>c M$ cannot hold with an absolute constant $c$.

Acknowledgement. We would like to thank to I. Ruzsa for his heipful comments.

References

[1] A. Balog and A. Sárközy, On sums of sequences of integers, II, Acta Math. Hung. 44 (1984), 169-179.

[2] J. Baumgartner, A short proof of Hindman's theorem, J.Comb. Th. Ser. A 17 (1974), 384-386.

[3] D. Berend, Joint ergodicity and mixing, J. Anaiyse Math. 45 (1985), 255-284.

[4] P. Erdös, Some unsolved problems, MTA MKI Közl. 6 (1961), 221-254.

[5] P. Erdös and Sárkōzy, On differences and sums of integers, I, J. Number Theory 10 (1978), 430-450.

[6] P. Erdős and A. Sárközy, On monochromatic products, to appear.

[7] H. Fürstenberg, Ergodic behavior of diagonal measures and a theorem of Szemerédi on arithmetic progressions, J. Analyse Math. 31 (1977), 204256.

[8] H. Fürstenberg and B. Weiss, Topological dynamics and combinatorial number theory, J. Analyse Math. 34 (1978), 61-85.

[9] R.K. Guy, Unsolved Problems in Number Theory, Springer-Verlag, 1981.

[10] D. Hilbert, Über die Irreduzibilität ganzer rationaler Functionen mit ganzzahligen Koefficienten, J. Reine Angew. Math. 110 (1892), 104-129.

[11] N. Hindman, Finite sums from sequences within cells of a partition of $\mathrm{N}$, J. Comb. Th. Ser. A 17 (1974), I-II. 
[12] J.C. Lagarias, A.M. Odlyzko and J.B. Shearer, On the Density of Sequences of Integers the Sum of No Two of which is a Square, I, Arithmetic Progressions, J. Comó. Th. Ser. A 33 (1982), 167-185.

[13] J.C. Lagarias, A.M. Odlyzko and J.B. Shearer, On the Density of Sequences of Integers the Sum of No Two of which is a Square, II, General Sequences, J. Comb. Th. Ser. A 34 (1983), 123-139.

[14] C. Pomerance, A. Sárkōzy and C.L. Stewart, On divisors of sums of integers, II, Pacific J. Math., to appear.

(15] I.Z. Ruzsa, oral communication.

[16] L.Z. Ruzsa, Probabilistic constructions in additive number theory, Soc. Math. France Astérique 147-148 (1987), 173-182.

[17] A. Sárközy, On difference sets of sequences of integers, I, Acta Math. Hung. 31 (1978), 125-149.

[18] A. Sárkōzy, On difference sets of sequences of integers, III, Acta Math. Hung. 31 (1978), 355-386.

[19] A. Sárkōzy and C.L. Stewart, On divisors of sums of integers, II, Math. Annalen.

[20] E. Szemerédi, On sets of integers containing no $k$ elements in arithmetic progression, Acta Arithm. 27 (1975), 199-245.

P. Erdos

Math. Inst. of the

Hung. Acad. of Sci.

Budapest
A. Sárközy

Math. Inst. of the

Hung. Acad. of Sci.

Budapest
V.T. Sós

Math. Inst. of the

Hung. Acad. of Sci.

Budapest 